\title{
Consensus Conditions for General Second-Order Multi-agent Systems with Communication Delay *
}

\author{
Wenying Hou ${ }^{\mathrm{a}}$, Minyue $\mathrm{Fu}^{\mathrm{b}, \mathrm{c}}$, Huanshui Zhang ${ }^{\mathrm{a}}$, Zongze $\mathrm{Wu}^{\mathrm{c}}$

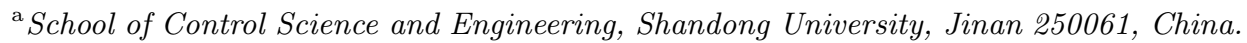 \\ ${ }^{\mathrm{b}}$ School of Electrical Engineering and Computer Science, University of Newcastle, NSW 2308, Australia. \\ ${ }^{\mathrm{c}}$ School of Automation, Guangdong University of Technology, \\ Guangdong Key Laboratory of IoT Information Technology, Guangzhou 510006, China.
}

\begin{abstract}
This paper studies the consensus problem for a class of general second-order multi-agent systems (MASs) with communication delay. We first consider the delay-free case and obtain a necessary and sufficient condition for consensus. Then, based on the obtained conditions for the delay-free case, we deduce an explicit formula for the delay margin of the consensus for the case with time delay using the relationship between the roots of the characteristic equation and the time delay parameter. In addition, we consider the special case where the second-order model is a double integrator. For this case, simpler consensus conditions under communication delay are provided.
\end{abstract}

Key words: Time-delay systems; multi-agent systems; frequency domain method; second-order consensus.

\section{Introduction}

Network consensus is a fundamental distributed control and optimization problem. After a couple of decades of active research on network consensus, it is well recognized by now that consensus control finds wide applications in areas including multi-agent coordination (such as coordinated decision making [2], vehicle formations [4], rendezvous problem [6], distributed computation [8], and flocking [13], et. al), smart electricity networks [9] and biological group behavioural analysis [18]. The key of consensus control is to design an appropriate consensus protocol based on local information exchange such that all the agents (or nodes) in a network agree upon certain quantities of common interest.

The pioneering work of [12] solved an average consensus problem for first-order integrator networks by using

\footnotetext{
‡ This paper was not presented at any IFAC meeting. This work is supported by the National Science Foundation of China under Grants 61120106011, 61573221.

* Corresponding author: Minyue Fu Tel. +61-408528333.

Email addresses: wyhou_00@163.com (Wenying Hou), Minyue.fu@newcastle.edu.au (Minyue Fu), hszhang@sdu.edu.cn (Huanshui Zhang), zzwu@scut.edu.cn (Zongze Wu).
}

the algebraic graph theory and frequency domain analysis. Since then, there has been a large number of results on consensus, e.g., [1], [4], [11], [14] and [15]. All of the above results on the first-order consensus problems focus on the first-order integrator systems or networks without time delay. However, the conditions that can guarantee consensus for the first-order MASs, for example, the network communication topology has a directed spanning tree, may not ensure the second-order MASs to reach consensus. In addition, in most applications, it is inevitable that time delay exists in the information transmission between agents due to communication congestion and finite transmission bandwidth. The existence of the communication delay will inevitably deteriorate the control performance and stability of a networked control system. Therefore it is important to consider consensus conditions of higher order MASs with communication delay.

Although there have been several papers studying the consensus problem with time delay, such as [19], [22], [20] and [3], they only focus on first-order consensus or they cannot give the explicit formula for the time delay margin for achieving consensus. Reference [10] considered time delay margin for unstable plants using frequency domain analysis. Second-order consensus problems can model more realistic dynamics of MASs. As 
far as the authors know, there are few papers considering the consensus problem for general second-order dynamic systems with time delay. References [23] and [16] considered the second-order consensus problem but only focused on double integrator systems.

In this paper, we consider the consensus condition for a class of MASs which contain a general second-order linear dynamic model for each agent and involve communication delay between agents. We first obtain a necessary and sufficient condition for consensus for the delayfree case. Then, based on the obtained conditions for the delay-free case, we deduce an explicit formula for the delay margin of the consensus for the case with time delay by analyzing the relationship between the roots of characteristic equation and the time delay parameter. This leads to the realization that there exists a fundamental tradeoff between consensus performance and robustness to time-delay. We will also provide a more detailed analysis on the consensus condition for the important special case where each agent is a double integrator, and provide a simple and explicit expression for the time delay margin for this case.

\section{Problem Formulation}

\subsection{Algebraic Graph Theory Basics}

Some basic knowledge on algebraic graph theory is needed for this paper. A multi-agent system (or network) is assumed to have $N$ agents. The communication topology between agents is denoted by a graph $\mathcal{G}=\{\mathcal{V}, \mathcal{E}, \mathcal{A}\}$, where $\mathcal{V}=\{1,2, \cdots, N\}$ is the set of agents, $\mathcal{E} \subset\{(i, j): i, j \in \mathcal{V}\}$ is the edge set, and $\mathcal{A}=\left[a_{i j}\right] \in \mathbf{R}^{N \times N}$ is the so-called weighted adjacency matrix (or adjacency matrix, for short). Each edge $(i, j)$ denotes that agent $j$ obtains information from agent $i$. The neighbouring set $\mathcal{N}_{i}$ of agent $i$ is the set of the agents that can obtain information from agent $i$. The a nonnegative elements and $a_{i j}>0$ if and only if $i \in \mathcal{N}_{i}$. The adjacency matrix $\mathcal{A}=\left\{a_{i j}\right\}$ is such that each element $a_{i j}>0$ if $(i, j) \in \mathcal{E}$, or $a_{i j}=0$. The in-degree of agent $i$ is denoted by $d_{i}=\sum_{j \in \mathcal{N}_{i}} a_{i j}=\sum_{j=1}^{N} a_{i j}$ and the in-degree matrix $\mathcal{D}=\operatorname{diag}\left\{d_{1}, d_{2}, \ldots, d_{N}\right\}$. The Laplacian matrix $\mathcal{L}$ of $\mathcal{G}$ is defined by $\mathcal{L}=\mathcal{D}-\mathcal{A}$. Note that $a_{i j}=a_{j i}, \forall i, j \in \mathcal{V}$ if and only if $\mathcal{G}$ is an undirected graph. A spanning tree of a digraph is a directed tree formed by graph edges that connects all the nodes of the graph. It is well known that for an undirected graph, $\mathcal{L}$ is a symmetric, positive semi-definite matrix and all of its eigenvalues are non-negative. Note the special property that $\mathcal{L} \mathbf{1}_{N}=\mathbf{0}_{N}$. By denoting all the eigenvalues of $\mathcal{L}$ as $\lambda_{i}, i=1,2, \ldots, N$, some properties of the Laplacian matrix are recalled below [7].

Lemma 1 The Laplacian matrix $\mathcal{L}$ has a simple eigenvalue 0 and all the other eigenvalues have positive parts if and only if the directed network has a directed spanning tree. Specially, for an undirected connected graph, all the eigenvalues of $\mathcal{L}$ are real numbers and can be arranged as $0=\lambda_{1}<\lambda_{2} \leq \ldots \leq \lambda_{N}$.

We use the following notations and conventions in this paper: $R$ denotes the real number field; $\mathbf{1}_{m}$ denotes the $m$-dimensional column vector with all components $1 ; I_{m}$ denotes the $m$-dimensional identity matrix; $\mathbf{0}$ denotes the zero matrix of appropriate dimension; $\operatorname{Re}(\theta)$ and $\operatorname{Im}(\theta)$ are the real and imaginary parts of a complex number $\theta$, respectively.

\subsection{Consensus Protocol}

In this paper we consider the following general secondorder linear dynamic model for each agent $i \in \mathcal{V}$ :

$$
\begin{aligned}
& \dot{x}_{i}(t)=v_{i}(t), \\
& \dot{v}_{i}(t)=a x_{i}(t)+b v_{i}(t)+u_{i}(t),
\end{aligned}
$$

where $x_{i}(t) \in R$ is the position state, $v_{i}(t) \in R$ is the velocity state of the $i$ th agent. The initial condition of the agent $i$ refers to $\left(x_{i}(0), v_{i}(0)\right)$.

Remark 2 Apparently, (1) can be seen as $\ddot{x}_{i}-b \dot{x}_{i}-$ $a x_{i}=u_{i}$, which is a general second-order differential equation. Alternatively, it can be seen as $\dot{\bar{x}}_{i}=A \bar{x}_{i}+B u_{i}$ with $\bar{x}_{i}=\left[x_{i}, v_{i}\right]^{T}, A=\left[\begin{array}{ll}0 & 1 \\ a & b\end{array}\right], B=\left[\begin{array}{l}0 \\ 1\end{array}\right]$, which is the general case of controllable canonical form of secondorder dynamics.

Definition 1 (second-order consensus) A multi-agent system $\mathcal{G}$ with agent model (1) is said to achieve secondorder consensus if, for any initial conditions and $i \neq j$, $i, j=1,2, \ldots, N$,

$\lim _{t \rightarrow \infty}\left\|x_{i}(t)-x_{j}(t)\right\|=0, \quad \lim _{t \rightarrow \infty}\left\|v_{i}(t)-v_{j}(t)\right\|=0$.

\section{Consensus Analysis for the Delay-Free Case}

Firstly, we deploy a control protocol without considering the time delay, which is given by

$$
\begin{aligned}
u_{i}(t)= & k_{1} \sum_{j=1}^{N} a_{i j}\left[x_{j}(t)-x_{i}(t)\right] \\
& +k_{2} \sum_{j=1}^{N} a_{i j}\left[v_{j}(t)-v_{i}(t)\right]
\end{aligned}
$$

where $k_{1} \in R$ and $k_{2} \in R$ are gain coefficients. We define the (composite) state vector $z(t)=\left[x^{T}(t), v^{T}(t)\right]^{T}$ 
with the (composite) position vector and velocity vector $x(t)=\left[x_{1}(t), x_{2}(t), \ldots, x_{N}(t)\right]^{T}, v(t)=$ $\left[v_{1}(t), v_{2}(t), \ldots, v_{N}(t)\right]^{T}$, respectively. The dynamics for the MAS are given by

$\dot{z}(t)=\Phi z(t)$,

where $\Phi=\left[\begin{array}{cc}0 & I_{N} \\ a I_{N}-k_{1} \mathcal{L} & b I_{N}-k_{2} \mathcal{L}\end{array}\right]$. Define $\hat{x}_{i}(t)=$ $x_{i}(t)-x_{1}(t), \hat{v}_{i}(t)=v_{i}(t)-v_{1}(t), i=2,3, \ldots, N$, and the state error vector as $\hat{z}(t)=\left[\hat{x}^{T}(t), \hat{v}^{T}(t)\right]^{T}$ with $\hat{x}(t)=$ $\left[\hat{x}_{2}(t), \hat{x}_{3}(t), \ldots, \hat{x}_{N}(t)\right]^{T}, \hat{v}(t)=\left[\hat{v}_{2}(t), \hat{v}_{3}(t), \ldots, \hat{v}_{N}(t)\right]^{T}$. We obtain the following error dynamics:

$\dot{\hat{z}}(t)=\hat{\Phi} \hat{z}(t)$,

where $\hat{\Phi}=\left[\begin{array}{cc}\mathbf{0} & I_{N-1} \\ a I_{N-1}-k_{1} \hat{\mathcal{L}} & b I_{N-1}-k_{2} \hat{\mathcal{L}}\end{array}\right]$, with $\hat{\mathcal{L}}=$ $L_{22}+\mathbf{1}_{N-1} \alpha^{T}$, and

$L_{22}=\left[\begin{array}{cccc}d_{2} & -a_{23} & \cdots & -a_{2 N} \\ -a_{32} & d_{3} & \cdots & -a_{3 N} \\ \vdots & \vdots & \ddots & \vdots \\ -a_{N 2} & -a_{N 3} & \cdots & d_{N}\end{array}\right], \quad \alpha=\left[\begin{array}{c}a_{12} \\ a_{13} \\ \vdots \\ a_{1 N}\end{array}\right]$

Apparently, system (1) or (3) achieves consensus if and only if the error system (4) is asymptotically stable.

Let $\beta=\left[a_{21}, a_{31}, \ldots, a_{N 1}\right]^{T}$, then $\mathcal{L}=\left[\begin{array}{cc}d_{1} & -\alpha^{T} \\ -\beta & L_{22}\end{array}\right]$.

Taking the transformation matrix $S=\left[\begin{array}{cc}1 & \mathbf{0}_{N-1}^{T} \\ \mathbf{1}_{N-1} & I_{N-1}\end{array}\right]$, then we have

$S^{-1} \mathcal{L} S=\left[\begin{array}{cc}0 & -\alpha^{T} \\ \mathbf{0}_{N-1} & \hat{\mathcal{L}}\end{array}\right]$

From (5) we can see that the eigenvalues of $\hat{\mathcal{L}}$ are $\lambda_{2}$, $\lambda_{3}, \ldots, \lambda_{N}$. In order to analyze the asymptotical stability of system (4), we consider its characteristic equation, i.e.,

$$
\operatorname{det}\left(s I_{2(N-1)}-\hat{\Phi}\right)=\prod_{i=2}^{N} f_{i}(s)=0
$$

where

$$
f_{i}(s)=s^{2}-b s-a+\left(k_{2} s+k_{1}\right) \lambda_{i} .
$$

We obtain the following result.
Lemma 3 The control protocol (2) makes the $M A S \mathcal{G}$ with (1) achieve consensus if and only if all $f_{i}(s), i=$ $2,3, \ldots, N$, are Hurwitz stable (i.e., their roots all have a negative real part).

Next we cite a frequency domain test for the stability of a polynomial [22].

Lemma 4 Given a polynomial $f(s)$, let $m(\omega)$ and $n(\omega)$ be the real and imaginary parts of $f(\iota \omega)\left(\iota^{2}=-1\right)$, respectively. Then $f(s)$ is Hurwitz stable if and only if $m(0) n^{\prime}(0)-m^{\prime}(0) n(0)>0$, and the polynomial pair $(m(\omega), n(\omega))$ is interlaced (i.e., $m(\omega)$ and $n(\omega)$ cross zero alternately as $\omega$ traverses from $-\infty$ to $+\infty)$.

Based on Lemmas 1, 3 and 4, we obtain the following consensus conditions for the delay-free case.

Theorem 5 The control protocol (2) makes the $M A S \mathcal{G}$ with (1) achieve consensus if and only if the following inequalities for $k_{1}$ and $k_{2}$ hold simultaneously for $i=$ $2,3, \ldots, N$ :

$$
\begin{aligned}
& k_{2}^{2} \operatorname{Im}^{2}\left(\lambda_{i}\right)-4\left[a-k_{1} \operatorname{Re}\left(\lambda_{i}\right)\right]>0, \\
& \frac{k_{1}^{2} \operatorname{Im}^{2}\left(\lambda_{i}\right)}{\left[b-k_{2} \operatorname{Re}\left(\lambda_{i}\right)\right]^{2}}+\frac{k_{1} k_{2} \operatorname{Im}^{2}\left(\lambda_{i}\right)}{b-k_{2} \operatorname{Re}\left(\lambda_{i}\right)}<k_{1} \operatorname{Re}\left(\lambda_{i}\right)-a, \\
& a b-\left(a k_{2}+b k_{1}\right) \operatorname{Re}\left(\lambda_{i}\right)+k_{1} k_{2}\left|\lambda_{i}\right|^{2}>0, \\
& b-k_{2} \operatorname{Re}\left(\lambda_{i}\right) \neq 0,
\end{aligned}
$$

where $\lambda_{i}, i=2,3, \ldots, N$, are the nonzero eigenvalues of the Laplacian matrix $\mathcal{L}$ and $\left|\lambda_{i}\right|$ is the module of $\lambda_{i}$.

PROOF. We apply Lemma 4 to Lemma 3 to deduce the stability conditions for $f_{i}(s)$. Note that $f_{i}(\iota \omega)=-\omega^{2}-$ $\iota b \omega-a+\iota k_{2} \omega \operatorname{Re}\left(\lambda_{i}\right)+k_{1} \operatorname{Re}\left(\lambda_{i}\right)-k_{2} \omega \operatorname{Im}\left(\lambda_{i}\right)+\iota k_{1} \operatorname{Im}\left(\lambda_{i}\right)$, thus $m_{i}(\omega)=-\omega^{2}-a-k_{2} \omega \operatorname{Im}\left(\lambda_{i}\right)+k_{1} \operatorname{Re}\left(\lambda_{i}\right)$, $n_{i}(\omega)=-b \omega+k_{2} \omega \operatorname{Re}\left(\lambda_{i}\right)+k_{1} \operatorname{Im}\left(\lambda_{i}\right)$. Denoting $\Delta_{i}=k_{2}^{2} \operatorname{Im}^{2}\left(\lambda_{i}\right)-4\left[a-k_{1} \operatorname{Re}\left(\lambda_{i}\right)\right]$, then the roots of $m_{i}(\omega)=0$ are given by

$$
u_{i 1}=\frac{-k_{2} \operatorname{Im}\left(\lambda_{i}\right)-\sqrt{\Delta_{i}}}{2}, \quad u_{i 2}=\frac{-k_{2} \operatorname{Im}\left(\lambda_{i}\right)+\sqrt{\Delta_{i}}}{2},
$$

and $n_{i}(\omega)=0$ has only one root, given by

$$
v_{i}=\frac{k_{1} \operatorname{Im}\left(\lambda_{i}\right)}{b-k_{2} \operatorname{Re}\left(\lambda_{i}\right)}
$$

Note that there must be $b-k_{2} \operatorname{Re}\left(\lambda_{i}\right) \neq 0$. And $m_{i}(0) n_{i}^{\prime}(0)-m_{i}^{\prime}(0) n_{i}(0)=a b-\left(a k_{2}+b k_{1}\right) \operatorname{Re}\left(\lambda_{i}\right)+$ $k_{1} k_{2}\left|\lambda_{i}\right|^{2}$. According to Lemma 4 , in order to guarantee the stability of $f_{i}(s)$, there must be

$$
\begin{aligned}
& \Delta_{i}>0, \quad u_{i 1}<v_{i}<u_{i 2}, \\
& m(0) n^{\prime}(0)-m^{\prime}(0) n(0)>0, \\
& b-k_{2} \operatorname{Re}\left(\lambda_{i}\right) \neq 0 .
\end{aligned}
$$


The condition (11) can be reduced to (7)-(10).

Remark 6 For an undirected connected graph, conditions (7)-(10) can be easily reduced to $k_{1}>a \lambda_{i}^{-1}$ and $k_{2}>b \lambda_{i}^{-1}, i=2,3, \ldots, N$.

If we let $a=b=0$, then system (1) becomes the following double integrator model:

$\dot{x}_{i}(t)=v_{i}(t), \quad \dot{v}_{i}(t)=u_{i}(t), \quad i=1,2, \ldots, N$.

We note that this model has been studied in the literature, as a non-trivial generalization of network consensus for first-order systems; see, e.g., [23] and [16]. It turns out that, by applying the protocol (2) and Theorem 4, we have the following result for this special case.

Corollary 7 The control protocol (2) makes the $M A S \mathcal{G}$ with (12) achieve consensus if and only if $\mathcal{G}$ has a directed spanning tree and the following inequalities for $k_{1}$ and $k_{2}$ hold:

$$
k_{1}>0, \quad k_{2}>0, \quad \frac{k_{2}^{2}}{k_{1}}>\max _{i=2,3, \ldots, N} \frac{\operatorname{Im}^{2}\left(\lambda_{i}\right)}{\operatorname{Re}\left(\lambda_{i}\right)\left|\lambda_{i}\right|^{2}} .
$$

PROOF. From Theorem 5 and $a=b=0$, we know that the control protocol (2) makes the MAS $\mathcal{G}$ with (12) achieve consensus if and only if the following inequalities of $k_{1}$ and $k_{2}$ hold simultaneously for $i=2,3, \ldots, N$ :

$$
\begin{aligned}
& k_{2}^{2} \operatorname{Im}^{2}\left(\lambda_{i}\right)+4 k_{1} \operatorname{Re}\left(\lambda_{i}\right)>0, \\
& k_{1}^{2} \operatorname{Im}^{2}\left(\lambda_{i}\right)<k_{1} k_{2}^{2} \operatorname{Re}\left(\lambda_{i}\right)\left|\lambda_{i}\right|^{2}, \\
& k_{1} k_{2}\left|\lambda_{i}\right|^{2}>0, \\
& -k_{2} \operatorname{Re}\left(\lambda_{i}\right) \neq 0,
\end{aligned}
$$

Apparently, from (14) and (17) we know that $\operatorname{Re}\left(\lambda_{i}\right) \neq 0$ or $\operatorname{Im}\left(\lambda_{i}\right) \neq 0, i=2,3, \ldots, N$, that is to say $\lambda_{i} \neq 0$, $i=2,3, \ldots, N$, so $\mathcal{L}$ has only one zero eigenvalue, and apparently all the other eigenvalues have positive parts. From Lemma 1, we know that $\mathcal{G}$ has a directed spanning tree.

From (16) we have $k_{1} k_{2}>0$, thus $k_{1}>0, k_{2}>0$ or $k_{1}<0, k_{2}<0$.

Case 1: $k_{1}>0, k_{2}>0$; Obviously, in this case, (14) and (17) hold for all $i=2,3, \ldots, N$, and (15) reduces to

$$
\frac{k_{2}^{2}}{k_{1}}>\frac{\operatorname{Im}^{2}\left(\lambda_{i}\right)}{\operatorname{Re}\left(\lambda_{i}\right)\left|\lambda_{i}\right|^{2}} .
$$

Case 2: $k_{1}<0, k_{2}<0$; Here (15) reduces to

$$
\frac{k_{2}^{2}}{k_{1}}>\frac{\operatorname{Im}^{2}\left(\lambda_{i}\right)}{\operatorname{Re}\left(\lambda_{i}\right)\left|\lambda_{i}\right|^{2}}
$$

Apparently, this is a contradiction.

From the above analysis, we know that only Case 1 can guarantee consensus, thus we have (13).

Remark 8 The result of Corollary 7 is consistent with references [23] and [16].

\section{Consensus Conditions with Constant Com- munication Delay}

In this section, we return to the case with communication delay $\tau$ and consider the following control protocol

$$
\begin{aligned}
u_{i}(t)= & k_{1} \sum_{j=1}^{N} a_{i j}\left[x_{j}(t-\tau)-x_{i}(t-\tau)\right] \\
& +k_{2} \sum_{j=1}^{N} a_{i j}\left[v_{j}(t-\tau)-v_{i}(t-\tau)\right] .
\end{aligned}
$$

Remark 9 We note in the control protocol above that the same delay $\tau$ also applies to node $i$. This is to ensure that correct error signals are used in the feedback to guarantee the consensusability. In applications where $x_{i}(t)$ is instantaneously known to node $i$, this signal needs to be delayed before being applied in $u_{i}(t)$. In applications where only relative information can be measured (e.g., $x_{i}(t)$ is not directly measured but only $x_{i}(t)-x_{j}(t)$ is measured) and time delay is involved in the measurement, taking the same time delay for node $i$ and node $j$ is natural. Note that relative measurements are common, including relative distance, relative velocity, etc.

Similarly, we have the following composite dynamics

$$
\dot{z}(t)=\left[\begin{array}{cc}
\mathbf{0} & I_{N} \\
a I_{N} & b I_{N}
\end{array}\right] z(t)-\left[\begin{array}{cc}
\mathbf{0} & \mathbf{0} \\
k_{1} \mathcal{L} & k_{2} \mathcal{L}
\end{array}\right] z(t-\tau),
$$

and the corresponding error dynamics

$$
\dot{\hat{z}}(t)=\left[\begin{array}{cc}
\mathbf{0} & I_{N-1} \\
a I_{N-1} & b I_{N-1}
\end{array}\right] \hat{z}(t)-\left[\begin{array}{cc}
\mathbf{0} & \mathbf{0} \\
k_{1} \hat{\mathcal{L}} & k_{2} \hat{\mathcal{L}}
\end{array}\right] \hat{z}(t-\tau)
$$

It is clear that the control protocol (18) makes the MAS $\mathcal{G}$ with (1) achieve consensus if and only if the error system (19) is asymptotically stable.

Similar to the delay-free case, we need to analyze the characteristic equation for the system (19). For this, we take the Laplace transform on (19) and obtain its char- 
acteristic equation as follows:

$$
\begin{aligned}
& \operatorname{det}\left[\begin{array}{c}
s I_{N-1} \\
-a I_{N-1}+e^{-\tau s} k_{1} \hat{\mathcal{L}}(s-b) I_{N-1}+e^{-\tau s} k_{2} \hat{\mathcal{L}}
\end{array}\right] \\
= & \prod_{i=2}^{N} f_{i}(s, \tau)=0
\end{aligned}
$$

where $f_{i}(s, \tau), i=2,3, \ldots, N$ are quasi-polynomials given by

$$
f_{i}(s, \tau)=s^{2}-b s-a+e^{-\tau s}\left(k_{2} s+k_{1}\right) \lambda_{i} .
$$

Then we obtain the following result.

Lemma 10 The control protocol (18) makes the MAS $\mathcal{G}$ with (1) and communication delay $\tau$ achieve consensus if and only if all the quasi-polynomials $f_{i}(s, \tau), i=$ $2,3, \ldots, N$ are Hurwitz stable (i.e., their roots all have a negative real part).

Similarly, for the special case of double integrator model (12), we have the following result.

Corollary 11 The control protocol (18) makes the MAS $\mathcal{G}$ with (12) and communication delay $\tau$ achieve consensus if and only if all the quasi-polynomials $s^{2}+e^{-\tau s}\left(k_{2} s+\right.$ $\left.k_{1}\right) \lambda_{i}=0, i=2,3, \ldots, N$ are Hurwitz stable.

By further analysis, we obtain the following result.

Theorem 12 Consider the $M A S \mathcal{G}$ with (1). Suppose that the control protocol (18) makes the MAS achieve consensus in the delay free case. For each $r \in\{2,3, \ldots, N\}$, let $\omega_{r}>0$ be the root of the following equation:

$$
\left(\omega_{r}^{2}+a\right)^{2}+b^{2} \omega_{r}^{2}-\left(k_{1}^{2}+k_{2}^{2} \omega_{r}^{2}\right)\left|\lambda_{r}\right|^{2}=0 .
$$

Take $\tau_{r}=\left\{k \pi+\arctan \Psi_{r}\right\} \omega_{r}^{-1}$, where

$\Psi_{r}=\frac{\phi_{r}\left(\omega_{r}^{2}+a\right)+\varphi_{r} b \omega_{r}}{\phi_{r} b \omega_{r}-\varphi_{r}\left(\omega_{r}^{2}+a\right)}$

with $\phi_{r}=k_{2} \omega_{r} \operatorname{Re}\left(\lambda_{r}\right)+k_{1} \operatorname{Im}\left(\lambda_{r}\right), \varphi_{r}=k_{2} \omega_{r} \operatorname{Im}\left(\lambda_{r}\right)-$ $k_{1} \operatorname{Re}\left(\lambda_{r}\right)$ and $k$ is the minimum integer such that $\tau_{r}>0$. Set $\tau^{*}=\min _{r} \tau_{r}$ (over all roots $\omega_{r}>0$ ). Then the control protocol (18) makes (1) achieve consensus if and only if $\tau \in\left[0, \tau^{*}\right)$.

PROOF. From Corollary 2.4 of [17] we know that for a quasi-polynomial of the form $f\left(s, e^{-\tau s}\right)=f_{0}(s)+$ $f_{1}(s) e^{-\tau s}$ with $f_{0}(s)=s^{n}+a_{1} s^{n-1}+\ldots+a_{n}, f_{1}(s)=$ $b_{1} s^{n-1}+\ldots+b_{n}$, if $f\left(s, e^{-\tau s}\right)$ is Hurwitz stable for $\tau=0$ and $f\left(s, e^{-\tau s}\right)$ is unstable for some $\tau>0$, then there must exist some $0<\tau^{*}<\tau$ such that $f\left(s, e^{-\tau^{*} s}\right)$ has a root on the imaginary axis and that $f\left(s, e^{-\tau^{0} s}\right)$ is stable for all $\tau^{0}<\tau^{*}$. Here since for $\tau=0$ the MAS achieves consensus, i.e., the quasi-polynomial of (20) is Hurwitz stable, thus the roots of (20) will still be in the open left half-plane for all $\tau \in\left(0, \tau^{*}\right)$ if and only if at least one of the quasi-polynomials $f_{i}\left(s, \tau^{*}\right), i=2,3, \ldots, N$ has imaginary roots. Next, we will only need to examine the imaginary roots of the quasi-polynomials of (20) for $\tau=\tau^{*}$.

Let $s_{r}=\iota \omega_{r}, \omega_{r} \in R, \omega_{r} \neq 0, r \in\{2,3, \ldots, N\}$. Then $f_{r}\left(s_{r}, \tau\right)=0$ means both of its real and imaginary parts are zero, which are given by $-\omega_{r}^{2}-$ $a+\phi_{r} \sin \left(\tau_{r} \omega_{r}\right)-\varphi_{r} \cos \left(\tau_{r} \omega_{r}\right)=0$, and $-b \omega_{r}+$ $\varphi_{r} \sin \left(\tau_{r} \omega_{r}\right)+\phi_{r} \cos \left(\tau_{r} \omega_{r}\right)=0$. Re-arranging the above gives $\sin \left(\tau_{r} \omega_{r}\right)=\left[\left(k_{1}^{2}+k_{2}^{2} \omega_{r}^{2}\right)\left|\lambda_{r}\right|^{2}\right]^{-1}\left[\phi_{r}\left(\omega_{r}^{2}+a\right)+\right.$ $\left.\varphi_{r} b \omega_{r}\right]$ and $\cos \left(\tau_{r} \omega_{r}\right)=\left[\left(k_{1}^{2}+k_{2}^{2} \omega_{r}^{2}\right)\left|\lambda_{r}\right|^{2}\right]^{-1}\left[\phi_{r} b \omega_{r}-\right.$ $\left.\varphi_{r}\left(\omega_{r}^{2}+a\right)\right]$. Form $\sin ^{2}\left(\tau_{r} \omega_{r}\right)+\cos ^{2}\left(\tau_{r} \omega_{r}\right)=1$ we can obtain that

$$
\left(\omega_{r}^{2}+a\right)^{2}+b^{2} \omega_{r}^{2}-\left(k_{1}^{2}+k_{2}^{2} \omega_{r}^{2}\right)\left|\lambda_{r}\right|^{2}=0 .
$$

Also we can obtain that $\tan \left(\tau_{r} \omega_{r}\right)=\Psi_{r}$, which yields $\tau_{r}=\frac{\arctan \Psi_{r}+k \pi}{\omega_{r}}$, where $k$ is an appropriate integer such that $\tau_{r}>0$.

Case 1: $\operatorname{Im}\left(\lambda_{r}\right)=0$. In this case, $(21)$ becomes $\left(\omega_{r}^{2}+\right.$ $a)^{2}+b^{2} \omega_{r}^{2}-\left(k_{1}^{2}+k_{2}^{2} \omega_{r}^{2}\right) \lambda_{r}^{2}=0$, which has two realvalued roots, $w_{r 1}>0$ and $\omega_{r 2}=-\omega_{r 1}$. We only need to consider the positive root, i.e., $\omega_{r 1}>0$, because the imaginary roots for $f_{r}(s, \tau)$ form complex conjugate pairs. For a fixed $\lambda_{r}>0$, we have $\Psi_{r 1}=-\Psi_{r 2}$. Thus $\omega_{r 1}^{-1} \arctan \Psi_{r 1}=\omega_{r 2}^{-1} \arctan \Psi_{r 2}$. So we can simply take $\tau_{r 1}=\tau_{r 2}$. That is to say, for the case of $\operatorname{Im}\left(\lambda_{r}\right)=0$, we only need to consider the corresponding time delay $\tau_{r}$ for $\omega_{r}>0$.

Case $2: \operatorname{Im}\left(\lambda_{r}\right) \neq 0$. Then there must exist $\lambda_{l}=\operatorname{Re}\left(\lambda_{r}\right)-$ $\iota \operatorname{Im}\left(\lambda_{r}\right)$. Let $s_{l}=\iota \omega_{l}, \omega_{l} \neq 0, l \in\{2,3, \ldots, N\}$, be the pure imaginary root of $f_{i}(s, \tau)$ for $i=l$, then

$$
\left(\omega_{l}^{2}+a\right)^{2}+b^{2} \omega_{l}^{2}-\left(k_{1}^{2}+k_{2}^{2} \omega_{l}^{2}\right)\left|\lambda_{l}\right|^{2}=0 .
$$

It is obvious that if $\omega$ is a root of $(21)$, then $-\omega$ is also a root of (22), and vice versa. Let $\omega_{i 1}>0$ and $\omega_{i 2}=-\omega_{i 1}$ be the roots of $\left(\omega_{r}^{2}+a\right)^{2}+b^{2} \omega_{r}^{2}-\left(k_{1}^{2}+k_{2}^{2} \omega_{r}^{2}\right)\left|\lambda_{r}\right|^{2}=0$. Then we have $\omega_{r 1}=\omega_{l 1}$ and $\omega_{r 2}=\omega_{l 2}$, thus $\phi_{l 1}=-\phi_{r 2}$, $\varphi_{l 1}=\varphi_{r 2}, \phi_{l 2}=-\phi_{r 1}, \varphi_{l 2}=\varphi_{r 1}$. So we can obtain that $\Psi_{l 1}=-\Psi_{r 2}, \Psi_{l 2}=-\Psi_{r 1}$. Thus, $\tan \left(\tau_{l 1} \omega_{l 1}\right)=$ $-\tan \left(\tau_{r 2} \omega_{r 2}\right)$ and we can simply take $\tau_{l 1}=\tau_{r 2}$. Similarly, we have $\tau_{l 2}=\tau_{r 1}$. That is to say, for the case of $\operatorname{Im}\left(\lambda_{r}\right) \neq 0$, we still only need to consider the corresponding time delay $\tau_{r}$ for $\omega_{r}>0$.

Finally, the minimum value of $\tau$ which yields some $f_{r}(s, \tau)$ to have a purely imaginary root is thus given 
by $\tau^{*}=\min _{r} \tau_{r}$ over all possible roots $\omega_{r}>0$ and $r \in 2,3, \ldots, N$.

Remark 13 The contribution of Theorem 12 is that it presents a method for finding the maximum tolerable time delay for the general second-order consensus problem.

Similarly, based on Corollary 7, Corollary 11 and Theorem 12, for the special case of double integrator systems (i.e., $a=b=0$ ), we have the following result.

Theorem 14 Consider the $M A S \mathcal{G}$ with (12). Suppose $\mathcal{G}$ has a directed spanning tree and that the control protocol (18) makes the MAS achieve consensus in the delay free case. For each $r \in\{2,3, \ldots, N\}$, let

$$
\tau_{r}=\omega_{r}^{-1}\left\{k \pi+\arctan \left(-\frac{\phi_{r}}{\varphi_{r}}\right)\right\},
$$

with $\phi_{r}=k_{2} \omega_{r} \operatorname{Re}\left(\lambda_{r}\right)+k_{1} \operatorname{Im}\left(\lambda_{r}\right), \varphi_{r}=k_{2} \omega_{r} \operatorname{Im}\left(\lambda_{r}\right)-$ $k_{1} \operatorname{Re}\left(\lambda_{r}\right), \omega_{r}=\sqrt{\frac{k_{2}^{2}\left|\lambda_{r}\right|^{2}+\sqrt{k_{2}^{4}\left|\lambda_{r}\right|^{4}+4 k_{1}^{2}\left|\lambda_{r}\right|^{2}}}{2}}$, and $k$ is the minimum integer such that $\tau_{r}>0$. Set $\tau^{*}=\min _{r} \tau_{r}$ (over all roots $\omega_{r}>0$ ). Then the control protocol (18) makes (1) achieve consensus if and only if $\tau \in\left[0, \tau^{*}\right)$.

Remark 15 Theorem 14 presents an explicit formula of the delay margin for second-order integrator consensus problem.

\section{Simulation Example}

In this section, we demonstrate our result through an example. We assume that there are five agents in a MAS. The adjacency matrix and the corresponding Laplacian matrix are

$\mathcal{A}=\left[\begin{array}{lllll}0 & 0 & 0 & 0 & 2 \\ 3 & 0 & 0 & 0 & 0 \\ 1 & 0 & 0 & 0 & 0 \\ 0 & 0 & 2 & 0 & 0 \\ 0 & 4 & 0 & 0 & 0\end{array}\right], \quad \mathcal{L}=\left[\begin{array}{rrrrr}2 & 0 & 0 & 0 & -2 \\ -3 & 3 & 0 & 0 & 0 \\ -1 & 0 & 1 & 0 & 0 \\ 0 & 0 & -2 & 2 & 0 \\ 0 & -4 & 0 & 0 & 4\end{array}\right]$

respectively. By calculation we know that $\lambda_{2}=1, \lambda_{3}=$ $2, \lambda_{4}=4.5+\frac{\sqrt{23}}{2} \iota, \lambda_{5}=4.5-\frac{\sqrt{23}}{2} \iota$. The second-order system model for each agent $i$ is given by

$$
\dot{x}_{i}(t)=v_{i}(t), \quad \dot{v}_{i}(t)=2 x_{i}(t)+v_{i}(t)+u_{i}(t),
$$

and the consensus protocol is given by (18). Firstly, from Theorem 5 we know that we can take $k_{1}=k_{2}=3$ to achieve consensus for the delay-free case. Based on these parameters and using Theorem 12, we compute that $\tau_{2}=0.4160, \tau_{3}=0.2176, \tau_{4}=0.1272, \tau_{5}=0.0629$. Thus $\tau^{*}=\min \left\{\tau_{2}, \tau_{3}, \tau_{4}, \tau_{5}\right\}=0.0629$. Hence, this MAS can reach consensus under the control protocol (18) if and only if $\tau<\tau^{*}$. Simulations for the error dynamics of the MAS for $\tau=0.06<\tau^{*}$ and $\tau=0.07>\tau^{*}$ can be displayed in Fig. 1 and Fig. 2, respectively. Apparently, the simulation results are consistent with Theorem 12.

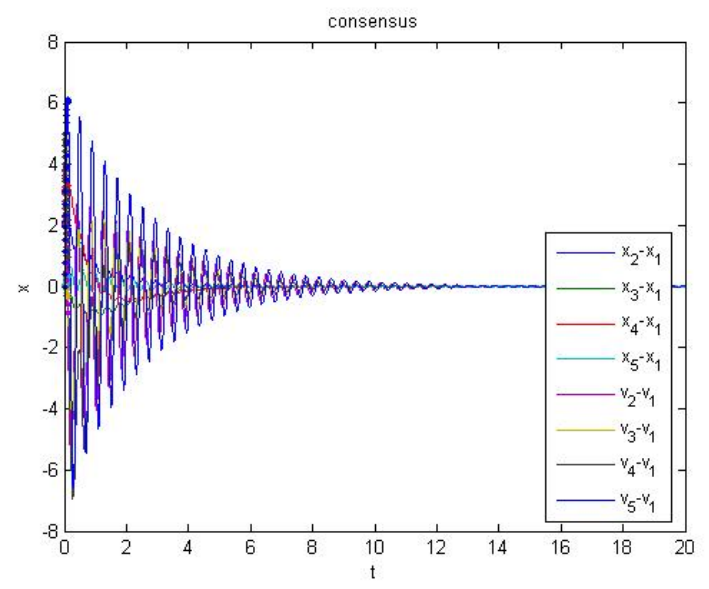

Fig. 1. $\tau=0.06<\tau^{*}$

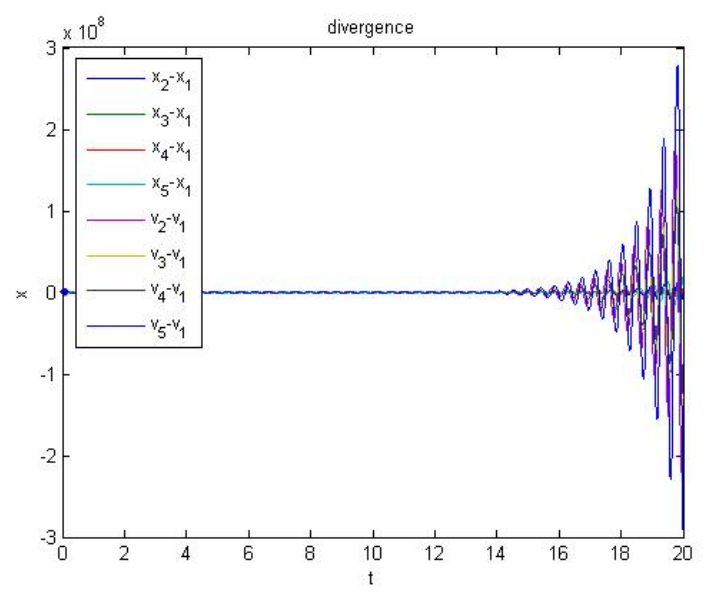

Fig. 2. $\tau=0.07>\tau^{*}$

Apparently, we can still take $k_{1}=k_{2}=3$ to guarantee consensus for the case of delay-free for double-integrator dynamics. Similarly, we can obtain that the critical delay is $\tau^{*}=0.0663$. The corresponding simulations of for $\tau=0.06<\tau^{*}$ and $\tau=0.07>\tau^{*}$ can be displayed in Fig. 3 and Fig. 4, respectively. The simulation results are consistent with Theorem 14 .

\section{Conclusion}

In this paper we have studied the consensus conditions for second-order linear MASs with communication delay. We first design a consensus-reaching control protocol for the delay-free case. This is then generalized to give an 


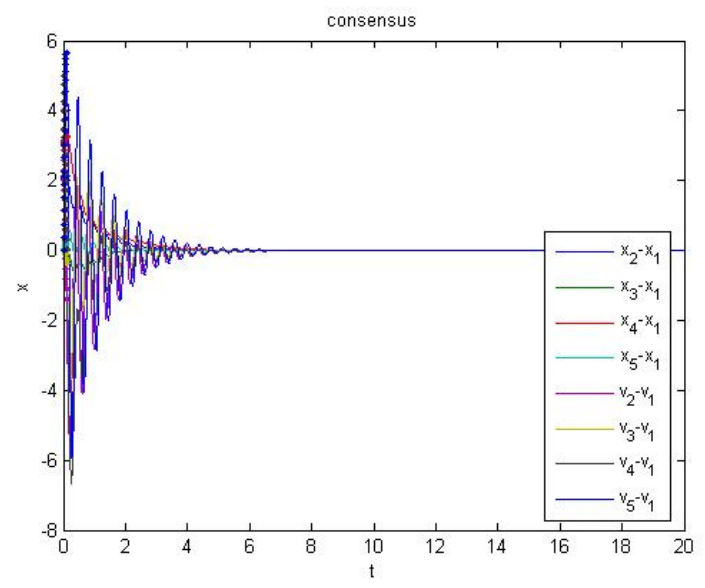

Fig. 3. $\tau=0.06<\tau^{*}$

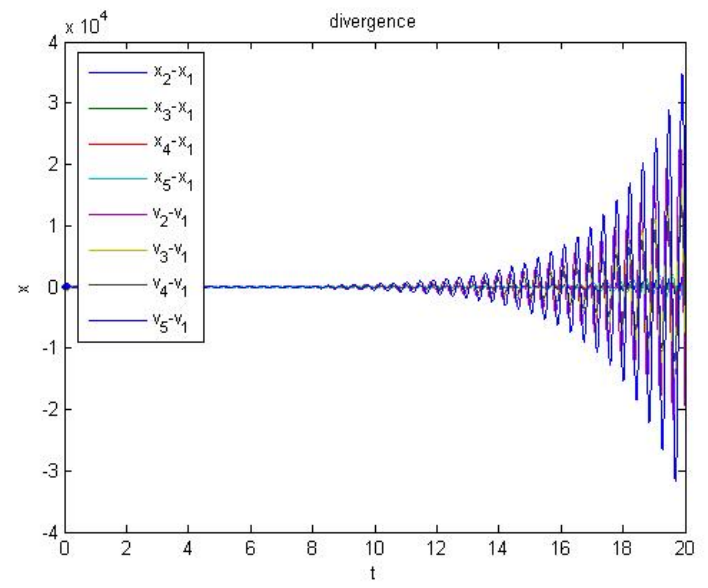

Fig. 4. $\tau=0.07>\tau^{*}$

explicit formula for the delay margin of the consensus for the case with time delay. In addition, we consider the special second-order linear MASs with double integrator models and provide explicit conditions for consensus. Future studies will focus on generalizing our results to higher order MASs.

\section{References}

[1] Avrachenkov, K., Chamie, M. E., \& Neglia, G. (2011). A local average consensus algorithm for wireless sensor networks, In Proc. of Distributed Computing in Sensor Systems and Workshops (DCOSS) (pp. 1-6).

[2] Bauso, D., Giarre, L., \& Pesenti, R. (2003). Distributed consensus protocols for coordinating buyers, In Proc. of Conference on Decision and Control (CDC) (pp. 588-592).

[3] Hou, W., Fu, M., \& Zhang, H. (2016). Consensusability of linear multi-agent systems with time delay, International Journal of Robust and Nonlinear Control, 26(12), 2529-2541.

[4] Fax, J. A., \& Murray, R. M. (2004). Information flow and cooperative control of vehicle formations, IEEE Transactions on Automatic Control, 49(9), 1465-1476.
[5] Lin, Z., Broucke, M., \& Francis, B. (2004). Local control strategies for groups of mobile autonomous agents, IEEE Transactions on Automatic Control, 49(4), 622-629.

[6] Lin, J., Morse, A. S., \& Anderson, B. D. O. (2003). The multi-agent rendezvous problem, In Proc. of Conference on Decision and Control (CDC) (pp. 1508-1513).

[7] Lewis, F. L., Zhang, H., Hengstermovric, K., \& Das, A. (2014). Cooperative control of multi-agent systems: Optimal and adaptive design approaches, London: Springer-Verlag.

[8] Lynch, N. A. Distributed algorithms. (1997). San Mateo, CA: Morgan Kaufmann.

[9] Ma, R., Chen, H., Huang, Y., \& Meng, W. (2013). Smart grid communication: Its challenges and opportunities, IEEE Tractions on Smart Grid, 4(1), 36-46.

[10] Middleton, R. H., \& Miller, D. E. (2007). On the achievable delay margin using LTI control for unstable plants, IEEE Transactions on Automatic Control, 52(7), 1194-1207.

[11] Moreau, L. (2005). Stability of multi-agent systems with time-dependent communication links, IEEE Transactions on Automatic Control, 50(2), 169-182.

[12] Olfati-Saber, R., \& Murray., R. M. (2004). Consensus problem in networks of agents with switching topology and time-delays, IEEE Transactions on Automatic Control, 49(9), 1520-1533.

[13] Olfati-Saber, R. (2006). Flocking for multi-agent dynamic systems: Algorithms and theory, IEEE Transactions on Automatic Control, 51(3), 401-420.

[14] Olfati-Saber, R., Fax, J. A., \& Murray, R. M. (2007) Consensus and cooperation in networked multi-agent systems, In Proc. of the IEEE, 95(1), 215-233.

[15] Ren, W., \& Beard, R. W. (2005). Consensus seeking in multi-agent systems under dynamically changing interaction topologies, IEEE Transactions on Automatic Control, 50(5), 655-661.

[16] Ren, W., \& Atkins, E. (2007). Distributed multivehicle coordinated control via local information exchange, International Journal of Robust and Nonlinear Control, 17(10-11), 1002-1033.

[17] Ruan, S., \& Wei, J. (2003). On the zeros of transcendental functions with applications to stability of delay differential equations with two delays, Dynamics of Continuous Discrete and Implusive Systems Series A: Mathematical Analysis, 10(6), 863-874.

[18] Strogatz, S. H. (2001). Exploring complex networks, Nature, 410, 268-272.

[19] Wang, X., Saberi, A., Stoorvogel, A. A., Grip, H. F., \& Yang, T. (2013). Consensus in the network with uniform constant communication, Automatica, 49(8), 2461-2467.

[20] Wang, Z., Xu, J., \& Zhang, H. (2014). Consensusability of multi-agent systems with time-varying communication delay, Systems \& Control Letters, 65(2014), 37-42.

[21] Xie, D., \& Wang, S. (2012). Consensus of second-order discrete-time multi-agent systems with fixed topology, Journal of Mathematical Analysis and Applications, 387(1), 8-16.

[22] Xu, J., Zhang, H., \& Xie, L. (2013). Input delay margin for consensusability of multi-agent systems, Automatica, 49(6), 1816-1820.

[23] Yu, W., Chen, G., \& Cao, M. (2010). Some necessary and sufficient conditions for second-order consensus, Automatica, 46(6), 1089-1095. 